This document is the accepted manuscript version of the following article:

Overbeck, J., Borin Barin, G., Daniels, C., Perrin, M. L., Braun, O., Sun, Q., ... Calame, M. (2019). A universal 1ength-dependent vibrational mode in graphene nanoribbons. ACS Nano. https://doi.org/10.1021/acsnano.9b05817

\title{
A Universal Length-Dependent Vibrational Mode in Graphene Nanoribbons
}

\author{
Jan Overbeck, ${ }^{*, \dagger,+, \uparrow, @ ~ G a b r i e l a ~ B o r i n ~ B a r i n, ~}{ }^{\dagger} @$ Colin Daniels, ${ }^{\S} @$ Mickael L. \\ Perrin, $^{\dagger}$ Oliver Braun, ${ }^{\dagger}, \ddagger$ Qiang Sun, ${ }^{\dagger}$ Rimah Darawish, ${ }^{\dagger, \|}$ Marta De Luca, ${ }^{\ddagger}$ \\ Xiao-Ye Wang, ${ }^{\perp, \triangle}$ Tim Dumslaff, ${ }^{\perp}$ Akimitsu Narita, ${ }^{\perp}$ Klaus Müllen, ${ }^{\perp, \#}$ Pascal \\ Ruffieux, ${ }^{\dagger}$ Vincent Meunier, ${ }^{*, \S}$ Roman Fasel, ${ }^{*}, \dagger, \|$ and Michel Calame,,,,$++ \uparrow$ \\ $\dagger$ †mpa, Swiss Federal Laboratories for Materials Science and Technology, 8600 Dübendorf, \\ Switzerland \\ $\ddagger$ University of Basel, Department of Physics, 4056 Basel, Switzerland \\ IUniversity of Basel, Swiss Nanoscience Institute, 4056 Basel, Switzerland \\ $\S$ Rensselaer Polytechnic Institute, Department of Physics, Applied Physics, and \\ Astronomy, Troy, New York 12180, United States \\ || University of Bern, Department of Chemistry and Biochemistry, 3012 Bern, Switzerland \\ $\perp$ Max Planck Institute for Polymer Research, 55128 Mainz, Germany \\ \#Institute of Physical Chemistry, Johannes Gutenberg-Universität Mainz, 55128 Mainz, \\ Germany \\ @ These authors contributed equally. \\ $\triangle$ Current address: State Key Laboratory of Elemento-Organic Chemistry, College of \\ Chemistry, Nankai University, Tianjin 300071, China \\ E-mail: jan.overbeck@empa.ch; meuniv@rpi.edu; roman.fasel@empa.ch; michel.calame@empa.ch
}




\section{Abstract}

Graphene nanoribbons (GNRs) have attracted considerable interest as their atomically tunable structure makes them promising candidates for future electronic devices. However, obtaining detailed information about the length of GNRs has been challenging and typically relies on low-temperature scanning tunneling microscopy. Such methods are ill-suited for practical device application and characterization. In contrast, Raman spectroscopy is a sensitive method for the characterization of GNRs, in particular for investigating their width and structure. Here, we report on a length-dependent, Raman active low-energy vibrational mode that is present in atomically precise, bottom-up synthesized armchair graphene nanoribbons (AGNRs). Our Raman study demonstrates that this mode is present in all families of AGNRs and provides information on their length. Our spectroscopic findings are corroborated by scanning tunneling microscopy images and supported by first-principles calculations that allow us to attribute this mode to a longitudinal acoustic phonon. Finally, we show that this mode is a sensitive probe for the overall structural integrity of the ribbons and their interaction with technologically relevant substrates.

\section{Keywords}

graphene nanoribbons, Raman spectroscopy, length-dependent mode, STM, substrate transfer, vibrational modes, DFT

Atomically precise graphene nanoribbons (GNRs) hold the promise of engineering the electronic properties of $\mathrm{sp}^{2}$-carbon systems over a wide range. ${ }^{1-3}$ While graphene has several electronic properties that make it an attractive material in certain applications, it lacks an electronic band gap, which limits its use for switching and optoelectronic applications. ${ }^{4}$ 
Lateral confinement of charge carriers in narrow graphene nanoribbons is one approach to overcome this limitation. A major advantage of GNRs is that their electronic band structure can be tailored via their width and edge structure. GNRs with armchair edges (AGNRs), for instance, cover the full range from quasi-metallic to wide band gap semiconductors. Based on the number $p$ of carbon-dimer lines present across their width, AGNRs can be grouped into three families: $3 p$ (medium gap), $3 p+1$ (wide gap) and $3 p+2$ (quasi-metallic gap). ${ }^{56}$ Within each family, the band gap scales inversely with the GNR width. A fabrication approach that allows for atomic control of ribbon width and edge structure is therefore indispensable. Onsurface synthesis has emerged as the prime technique to fabricate atomically precise GNRs. Based on metal surface-assisted covalent coupling of specifically designed molecular precursors, this bottom-up approach has proven to be successful for the fabrication of GNRs with different widths (5-,7-,9-,13-AGNRs) and edge topologies (armchair-, zigzag-, chiral-GNRs and GNRs with topological phases).131710 In order to exploit GNRs for electronic devices, they need to be transferred from their metallic growth substrate onto a technologically suitable one $\stackrel{11112}{ }$ First experiments with GNRs incorporated into devices have shown that the ribbon length as well as their overall integrity are essential for realizing the inherent potential of this material. $\stackrel{13114}{ }$ However, methods suited to estimate the length of the GNRs after transfer onto device substrates are lacking.

Here, we report on a length-dependent vibrational mode in atomically precise AGNRs observed at small Raman shifts. This mode, identified as a longitudinal acoustic phonon by first-principle- calculations, is present in all families of AGNRs and is the first vibrational characteristic that provides information about the GNRs' length after transfer onto device substrates. We analyze in detail its properties based on a combined study via Raman spectroscopy, scanning tunneling microscopy (STM), and computational modeling. Finally, we show that the frequency of this mode is highly sensitive to substrate interaction and the presence of defects, making it ideally suited for the investigation of GNR integrity and damage monitoring. 
Due to its ease of use, efficacy, and sensitivity to structural details, Raman spectroscopy has emerged as a primary method for the investigation of GNRs, in particular after transfer onto insulating materials where STM characterization is not possible. Many of the Raman-active phonon modes in GNRs are named in analogy to the terminology used in graphite, graphene, and carbon nanotubes (CNTs). For example, the LO-/TO-modes are referred to as the G-peak and its properties have been the focus of several studies. $\frac{12|15| 16}{1 n}$ contrast to graphene and CNTs, additional phonon modes found in the spectral range of 1100-1500 $\mathrm{cm}^{-1}$ are not a sign of the presence of defects but rather a result of the (hydrogenpassivated) edges breaking the periodicity of a perfect honeycomb lattice. These features, referred collectively as making up the $\mathrm{CH} / \mathrm{D}$-region, can be used to identify and probe the edge structure of GNRs. $\frac{17118}{17}$ The fundamental transverse acoustic mode of GNRs, named radial breathing-like mode (RBLM) in reference to its counterpart in CNTs, is commonly used to identify the ribbon width. $\frac{16 / 17 / 19}{19}$ As the Raman shift, intensity, and peak widths associated with these modes depend on the GNR structure, they have been used to monitor

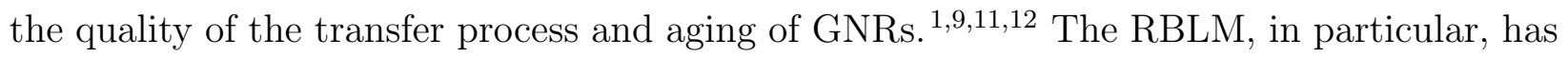
been used to monitor growth-related processes such as lateral fusion of GNRs. $\frac{20 \mid 21}{21}$

In the above-mentioned studies GNRs are treated as 1D-objects of quasi-infinite length, which allows modeling them in a periodic framework. ${ }^{5}$ This, however, is a highly idealized picture as can be seen from STM images showing significant amounts of short ribbons, already present on the growth substrate. ${ }^{2}$ As the GNRs' length is a crucial factor for their integration into functional devices, a universally applicable method for detecting and modelling finite size GNRs is highly desirable. In the following, we present such a method which we apply to 5-, 7- and 9-AGNRs, as distinct representatives of all three AGNR families. The GNRs are grown by on-surface synthesis on gold as described by Cai et al. and further transferred onto device substrates (see Methods for details). 
a)

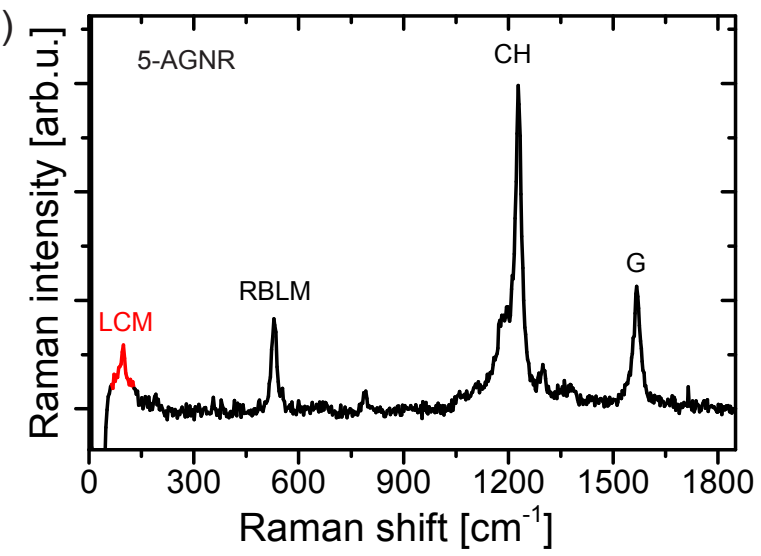

b)

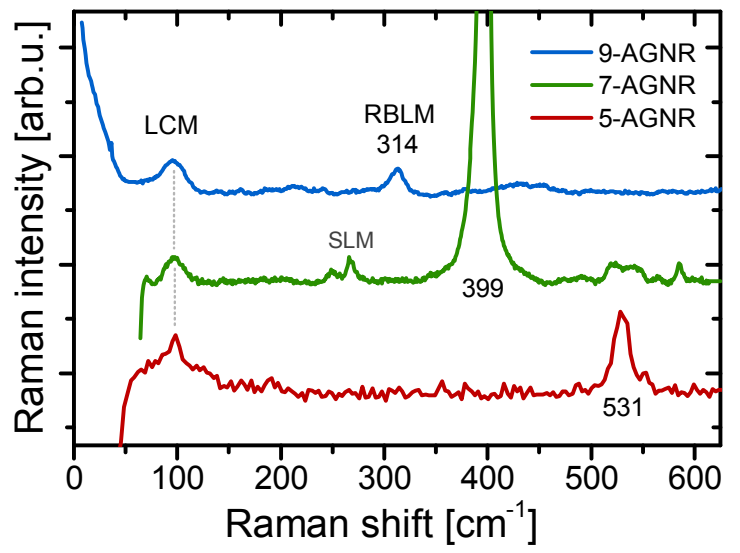

c) Longitudinal Compressive Mode (LCM)

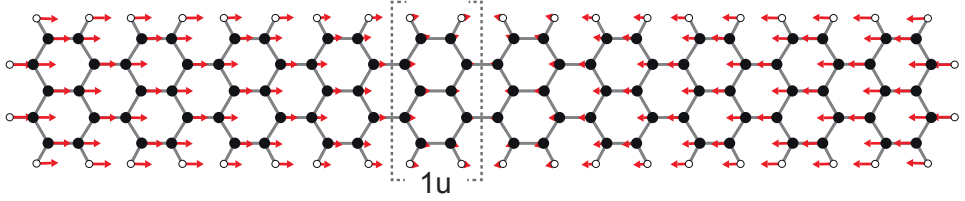

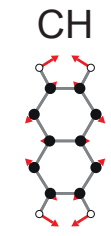

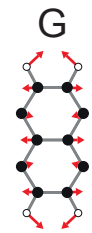

Figure 1: Longitudinal vibrational mode in armchair GNRs. a) Raman spectrum of 5-AGNRs with assignment of the most prominent peaks: G- \& CH- modes, RBLM and a low-energy peak at approximately $100 \mathrm{~cm}^{-1}$, labelled LCM. Excitation wavelength $\lambda_{\text {exc }}=$ 785 nm. Sample 5-1_Au, see Table S1, b) Low-energy Raman spectra of 5-, 7- and 9-AGNRs (offset for clarity), each exhibiting a peak slightly below $100 \mathrm{~cm}^{-1} . \lambda_{\text {exc }}=785 \mathrm{~nm}, 532 \mathrm{~nm}$ and $488 \mathrm{~nm}$ for Samples 5-1_Au, 7-1_T, 9-1_T, respectively. c) Atomic displacement profiles obtained from DFT calculations of 5-AGNRs matching the peaks in a). LCM for a finite-size 10-unit GNR. A single naphthalene unit is referred to as 1u. RBLM, CH- and G-modes are shown for a single unit-cell of a periodic ribbon. C-atoms are shown with filled, H-atoms with open circles.

\section{Results and Discussion}

A longitudinal compressive mode in GNRs. Figure 1 a) shows a Raman spectrum of 5-AGNRs obtained directly on the $\mathrm{Au}(788)$ growth substrate. The corresponding STM images are shown in Figure S1. Unless stated otherwise, Raman spectra are obtained in a homebuilt vacuum chamber using an optimized mapping approach to minimize damage to the GNRs, to obtain a high signal-to-noise ratio, and to probe the average properties of the GNR film (see Methods). ${ }^{22}$ In the spectrum we identify the G-, CH- and RBLM-peaks described above. In addition to these modes, we detect a low-energy peak slightly below $100 \mathrm{~cm}^{-1}$ (labeled LCM). This mode has, to the best of our knowledge, not been discussed 
for GNRs to-date and is the focus of this study. We argue below that it originates from a longitudinal vibration of the GNR and that it is extremely useful for GNR characterization and to probe their structural integrity upon transfer and device integration.

We first discuss the low-energy modes that have already been reported for armchair-type GNRs and how they can be distinguished from this additional mode. The lowest mode usually reported is the well-documented RBLM, the fundamental acoustic mode for which all atoms move in-plane in the direction perpendicular to the ribbon axis (see Figure 1c)]. It is observed at a Raman shift of about $534 \mathrm{~cm}^{-1}, 399 \mathrm{~cm}^{-1}$ and $313 \mathrm{~cm}^{-1}$ for the 5 -, 7 - and 9-AGNRs, respectively. Modes at energies below the RBLM of a particular GNR have been attributed to the formation of wider GNRs with correspondingly lower RBLM frequency by thermally-induced lateral fusion. $\frac{20121}{1}$ We can exclude this effect here as we do not reach the temperatures required to induce lateral fusion, nor do we observe them in STM or measure the characteristic series of RBLMs for GNRs of integer multiple widths of the fundamental ribbon. ${ }^{20}$ Another low-energy mode is the shear-like mode (SLM), which has been observed in 7-AGNRs and has recently been discussed for 5-AGNRs and 9-AGNRs. ${ }^{22 \mid 23}$

To clarify whether the LCM-mode at $100 \mathrm{~cm}^{-1}$ is specific to 5 -AGNRs or is a universal property of all AGNRs, we also acquired Raman spectra on 7-\&9-AGNRs. Figure 1 b) displays the low-energy spectra of 5-, 7-, and 9-AGNRs. In all spectra, a peak at roughly the same Raman shift is clearly identified, pointing towards the universal presence of this mode in AGNRs. While the spectrum for 5-AGNRs was acquired directly on the $\mathrm{Au}(788)$ growth substrate, the spectra for 7-\&9-AGNRs were acquired after transferring the GNRs to a device substrate optimized to enhance the Raman signal. ${ }^{22}$ For each type of GNR the wavelength and laser power were independently optimized to obtain the highest signal to noise ratio. The presence of this mode in multiple AGNRs, always at frequencies below their fundamental transverse acoustic mode (RBLM) points towards an even longer-range mode associated with a longitudinal acoustic vibration.

First-principles modeling of finite-size GNRs, in contrast to the usual approach of simu- 
lating infinitely long ribbons via periodically repeated unit cells, produces precisely such a longitudinal compressive mode (LCM) (see Figure 1c). The emergence of these modes stems directly from a zone-folding effect that accounts for the dimensionality reduction of a 1D periodic GNR into a 0D finite-size GNR. In fact, the LCM mode and its overtones arise from the quantization of $\mathrm{q}$ along the LA branch. In SI Note 2 we show a mapping of the phonon bandstructure from a 1D to finite-size ribbons of two different lengths. For a quantitative modeling of these low-energy modes, we performed Raman spectra calculations using both density functional theory (DFT) as well as separate calculations using a combination of the REBOII force-field and a bond polarizability model. The latter calculations were carried out in order to examine overall trends and larger systems not easily tractable by DFT. For details see Methods and SI Note 3.

Length-dependence and quantum mechanical calculations. In Figure 2 a), we initially compare the calculated Raman spectra for all three GNR widths for a length of about $12 \mathrm{~nm}$ (28 units). For these long GNRs, we employ the combined REBOII and bond polarizability method as Raman calculations at the DFT level are prohibitively computationally expensive.

The most prominent peak is the RBLM, which shows the well-known width-dependent Raman shift. $\frac{16|17| 19}{}$ Also, the position of the shear-like mode (SLM) mentioned earlier shows a clear down-shift with increasing GNR width. ${ }^{23}$ In contrast, the frequency of the LCM and its overtone does not change as a function of width of the three GNRs, matching our experimental observations in Figure $1 \mathrm{~b}$ ), This can be rationalized by the nearly constant ratio of mass per unit cell to the number of bonds along the GNR axis. In a simple mass-andspring approximation, this ratio determines the frequency of the normal mode considering the atomic displacement profile of the LCM along the GNR axis, as shown in Figure 11. and S5. It would therefore be interesting to investigate GNRs with armchair segments of different width to clarify the effect of changing the vibrating mass vs. ribbon stiffness. ${ }^{9[10]}$

The calculations show a strong change of the LCM frequency as a function of GNR length. 
a)



c)

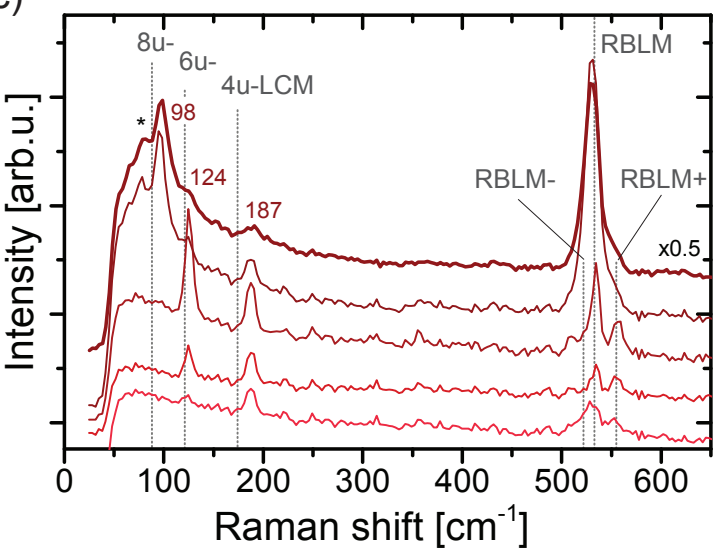

b)

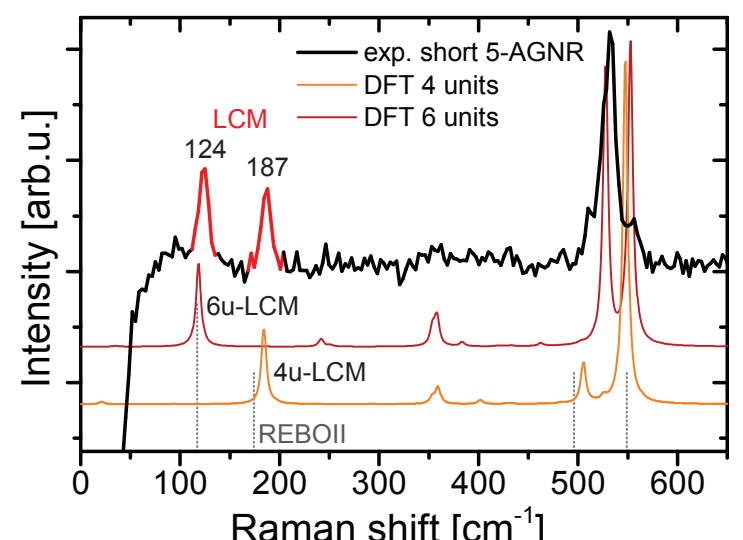

d)

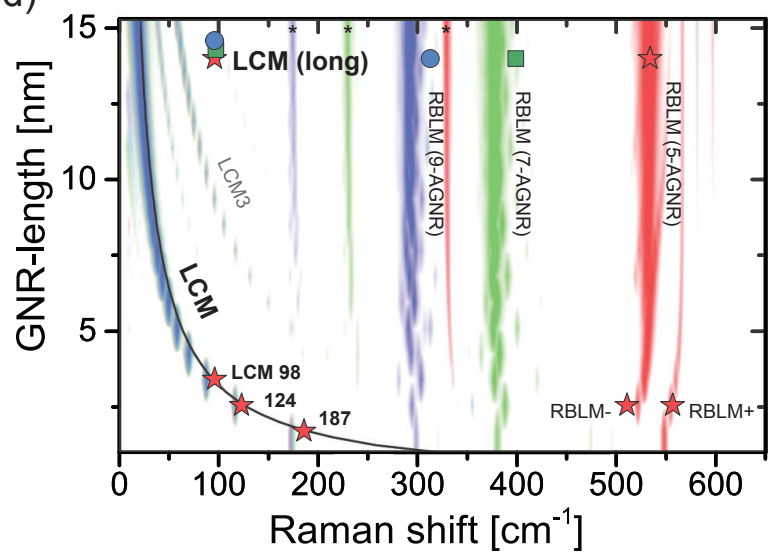

Figure 2: Length-dependence of GNR-modes in experiment and Raman calculations. a) REBOII-based spectra for long, finite (28 units $\approx 12 \mathrm{~nm}$ length) 9-, 7-, and 5-AGNRs and shorter 5-AGNRs. b) Experimental Raman spectrum for 5-AGNRs from a sample with high content of short ribbons (black line) and DFT-calculated spectra for 4- \& 6-unit $(4 \mathrm{u}, 6 \mathrm{u})$ length 5 -AGNRs. Dotted lines indicate the frequencies obtained from the REBOII-based calculations for the corresponding LCM modes. $\lambda_{\text {exc }}=785 \mathrm{~nm}$, Sample 56_Au. c) Raman spectra for a selection of 5-AGNR samples on Au substrates, offset for clarity. REBOII-calculated frequencies for the 4/6/8u-LCM and $6 \mathrm{u}-\mathrm{RBLM}^{+/-}$are indicated in grey. $\quad \lambda_{\text {exc }}=785 \mathrm{~nm}$, Samples $5-1$ Au to 5-5_Au, top to bottom. d) Map of REBOIIcalculated length-dependent spectral intensity, superimposed with the experimental mode frequencies (symbols) and analytical model (black line). Intensity values are color-coded on a logarithmic scale from white to red (green/blue) for 5-AGNRs (7-/9-AGNRs). Symbols are colour coded accordingly. The LCM for the three GNRs overlap, preventing distinction by color. Black asterisks indicate the shear-like modes (SLM).

In the lower part of Figure $2 \mathrm{a}$ ) we show three spectra of shorter 5-AGNRs that exhibit a progressive up-shift of the LCM as the ribbon gets shorter. To probe this effect experimentally, we fabricated a sample with an unusually high number of very short 5-AGNRs as determined from low temperature STM (LT-STM; see FigureS2). Short GNRs are usually 
a result of a premature hydrogen-passivation during the polymerization step, which is sensitive to the halogen-functionalization of the precursor molecules. ${ }^{24}$ It can also be caused by mono-bromo(iodine) functionalized molecules, which can be used to identify inappropriate growth conditions or inadequate precursor storage. The shortest ribbon segments obtained in our experiments with 5-AGNRs are 4-units and 6-units long, composed of two and three precursor molecules, respectively. Such short ribbons are extremely mobile at room temperature requiring low-temperature STM characterization in order to quantify the GNRs' length distribution (see Figure S2). Figure 2 b) displays the Raman spectrum of a sample with short 5-AGNRs, exhibiting two prominent peaks below $200 \mathrm{~cm}^{-1}$. Also plotted are the calculated Raman spectra for 4- and 6-unit 5-AGNRs, which can be treated entirely in DFT because of their limited size. The spectra match well with the peak positions observed experimentally for the LCM, without any need for frequency scaling. Moreover, the DFT-spectra also reproduce the side-peaks observed on the RBLM, which result from the splitting of the RBLM into normal modes with diagonal atomic displacement for these short ribbons (see Figure S5 for the atomic displacements). To assess the sensitivity of the Raman spectra on the computational method, we also calculated the LCM frequencies via forces calculated by the REBOII potential (dotted lines). The results are very similar, validating its use for longer GNRs. The results also indicate that the relevant REBOII-calculated forces tend to be weaker than those from DFT and thus the mode frequencies are slightly underestimated for these acoustic modes (also observed for the RBLM of 7-/9-AGNRs).

Next, we experimentally investigate the influence of the ribbon length. Figure 2c) shows Raman spectra obtained on several samples of 5-AGNRs with changing percentages of short ribbons tuned via the growth parameters. The spectra are sorted, from bottom to top, by increasing average GNR length as deduced from STM imaging. The bottom spectrum shows the peak at $187 \mathrm{~cm}^{-1}$ attributed to the $4 \mathrm{u}-\mathrm{LCM}$, as well as a small peak at $125 \mathrm{~cm}^{-1}$ where the $6 \mathrm{u}-\mathrm{LCM}$ is expected. For increasing average ribbon length, the relative amplitude of the $6 \mathrm{u}-\mathrm{LCM}$ becomes more prominent, whereas the 4u-LCM decreases. For even longer 
average ribbon length, a third peak appears centered at $98 \mathrm{~cm}^{-1}$, which we attribute to the $8 \mathrm{u}-\mathrm{LCM}$, while the peaks at $125 \mathrm{~cm}^{-1}$ and $187 \mathrm{~cm}^{-1}$ disappear. Notably, unassigned modes at comparable frequencies can be found in Raman spectra published by other groups. $\frac{2125}{2}$ The RBLM shows a similar trend with side peaks clearly visible for the same spectra that show the strongest signal of $4 \mathrm{u}-\& 6 \mathrm{u}-\mathrm{LCM}$. For the two topmost spectra, the central RBLM-peak characteristic of long GNRs is recovered.

Figure 2d) provides an overview of the theoretical and experimental data in a single plot. For the calculations, the length of the GNRs is varied from 4 units to 36 units $(1.7 \mathrm{~nm}$ to $15.3 \mathrm{~nm}$ ) along the y-axis. The computed Raman scattering intensities are represented on a logarithmic color-scale from white to red for 5-AGNRs, and from white to green/blue for 7-/9-AGNRs as a function of Raman shift (x-axis). As shown in Figure 2a), the RBLMs of the three GNRs are well separated and do not significantly change as a function of length, except for the splitting seen in very short 5-AGNRs. The LCM, in contrast, overlaps for the three different GNRs and shows a common frequency-downshift with increasing GNR length. This trend is also seen for its overtone, labeled LCM3 on the figure. The SLMs are marked by small asterisks and are essentially independent of length, as expected. The experimental data are superimposed as symbols with the same red/green/blue color-coding for 5-/7-/9-AGNRs, respectively. We display the experimental data at an assigned length of $14-15 \mathrm{~nm}$ for 'long' GNRs produced with the optimized growth parameters. This is a lower bound for the length distribution determined from STM images (see Figure S3 b)]. 24 The discrepancy in Raman shift of the LCM between experiment and theory for these long ribbons is discussed below. Finally, for the LCM we plot an analytical curve based on Equation 2 in SI Note 2, derived from a zone-folding approach. Gillen et al. used this to model the frequency of the RBLM as a function of width. The frequency $\omega_{\mathrm{LCM}}=\pi \cdot V / L$, where $L$ is the ribbon length and $V=1026 \AA^{-1} \mathrm{~cm}^{-1}$ is the speed of sound in graphene $e^{26}$, results in excellent agreement with our other calculations as well as our experiments. 

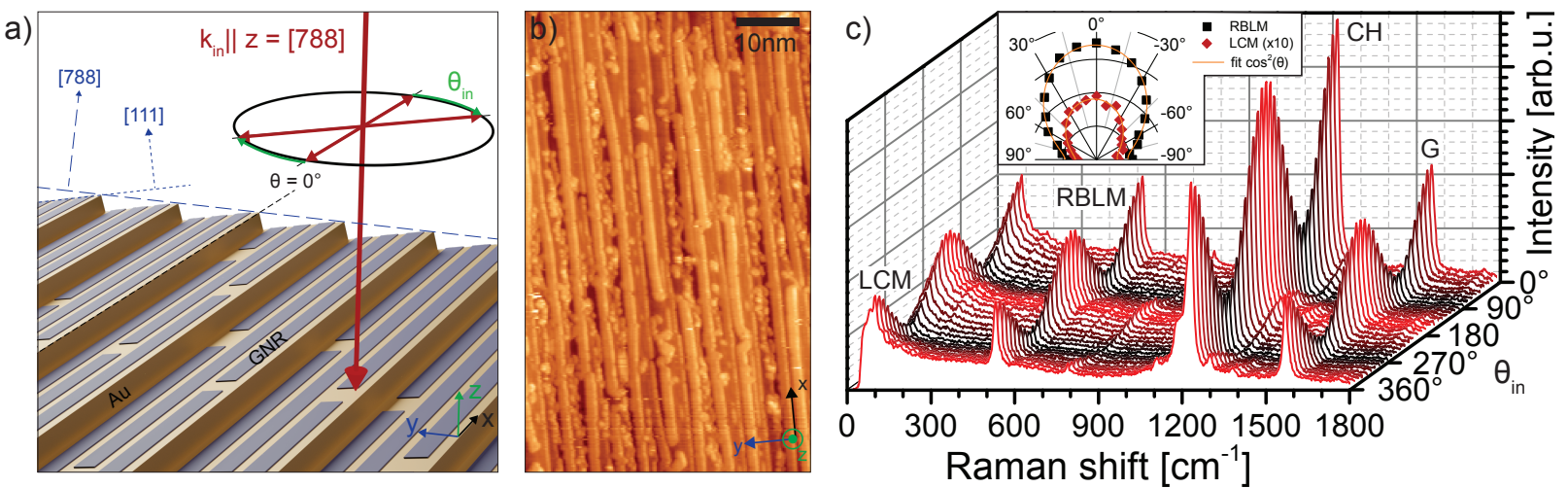

Figure 3: Characterization of the LCM for 5-AGNRs. a) Sketch of the measurement geometry for aligned GNRs grown on terraced $\mathrm{Au}(788)$ crystals. The incoming light under normal incidence to the $\mathrm{Au}(788)$ surface is polarized at an angle $\theta_{\text {in }}$ relative to the direction of the gold terraces. b) STM image of aligned GNRs (sparsely grown 9-AGNRs for the purpose of illustration). c) Raman spectra of aligned 5-AGNRs on a $\mathrm{Au}(788)$ growth substrate as a function of polarization of the exciting laser at $\lambda_{\text {exc }}=785 \mathrm{~nm}$. Sample 5-1_Au. Inset: polar plot of the RBLM \& LCM (scaled x10) mode intensities $I \propto \cos ^{2}(\theta)$.

Polarization analysis of the LCM. Polarization-dependent measurements provide additional information on the nature of Raman-active vibrations. As a consequence of their high aspect ratio, GNRs show a characteristic anisotropic dependence of scattering intensity on the relative alignment of light polarization direction and ribbon axis, an effect known from other quasi-1D materials such as carbon nanotubes. ${ }^{[27-31}$ We probe this dependence for 5-AGNRs grown on a $\mathrm{Au}(788)$ crystal where the narrow (3-4 nm) (111)-terraces favor unidirectional growth of 5-AGNRs parallel to the step edges separating the terraces (see Figure 3a, b) . To probe the global alignment of GNRs by Raman spectroscopy we vary the angle $\theta_{\text {in }}$ of the linear polarization of the excitation laser with respect to the direction of the terraces $\theta=0^{\circ}$ (see Figure $3 \mathrm{a}$ ) and SI-Note 3). No polarizer was used in the detection path to maximize the signal intensity. Figure 3c) shows a waterfall plot of Raman spectra of aligned 5-AGNRs as a function of polarization angle. The intensity of the low-energy LCM clearly follows that of the RBLM, CH- \& G-modes. The inset shows the peak intensities of the RBLM and LCM displayed in a polar plot. The behavior is well-described by an ensemble of dipoles, each with an $\mathrm{I}\left(\theta_{\text {in }}\right) \propto \cos ^{2}\left(\theta_{\text {in }}\right)$ characteristic, that are preferably oriented along 
the $\theta=0^{\circ}$-direction. ${ }^{31}$ The fact that all GNR modes, including the LCM, follow a common polarization dependence irrespective of intrinsic mode symmetry is attributed to the strong shape anisotropy. 29130 We use the polarization anisotropy $\mathrm{P}$ as a measure to quantify this effect and find a value of $\mathrm{P}_{\mathrm{LCM},(\mathrm{RBLM})}=0.5,(0.6)$ see SI Note 4 . The length of GNRs also affects the observed polarization-dependence. In Figure S11 we show measurements on samples containing predominantly short GNRs. We observe a lower polarization anisotropy $\mathrm{P}_{\text {RBLM }}=0.4$ than for the GNRs grown with optimized parameters (Figure $3 \mathrm{c}$ ). We attribute this to the combination of two effects: First, a lower degree of ribbon alignment as seen in STM because short ribbons are not restricted in growth direction by the width of the terraces, and second, a reduced shape-anisotropy as reflected in the optical absorption matrix elements. $\underline{30}$

Finally, we investigate the effect of transferring the GNRs from their gold growth substrate to standard silicon and Raman optimized device substrates. ${ }^{22}$ Figures S6, S8, and S9 of SI Note 3 show the LCM in spectra for the three types of AGNRs after transfer. Again, we performed polarization-dependent measurement on the LCM of aligned 7- and 9-AGNRs. As for aligned 5-AGNRs, the LCM follows the polarization dependent intensity of the other Raman active GNR modes (Figure S10). For 9-AGNRs with an average length of about $40 \mathrm{~nm}$ we observe a significantly larger polarization anisotropy $\left(\mathrm{P}_{\mathrm{LCM}}>0.9\right.$ and $\mathrm{P}_{\text {RBLM }}>0.7$ ). Samples of (globally) non-aligned 5-AGNRs synthesized on Au/Mica substrates with wider terraces, too, show the LCM and RBLM at the same frequencies but without any polarization dependence (see SI Note 1 and Figures S3 b) and S8 a) for STM and Raman spectra). Beyond confirming the ribbon-origin of the LCM, these polarizationdependent measurements can therefore be used as an independent, albeit rough, indication of GNR length for well-aligned ribbons. Altogether, for all three families of AGNRs, we find a common polarization dependence of the LCM and the other Raman modes, and that the transfer procedure does not significantly impact the GNR structure as revealed by its vibrational modes. 

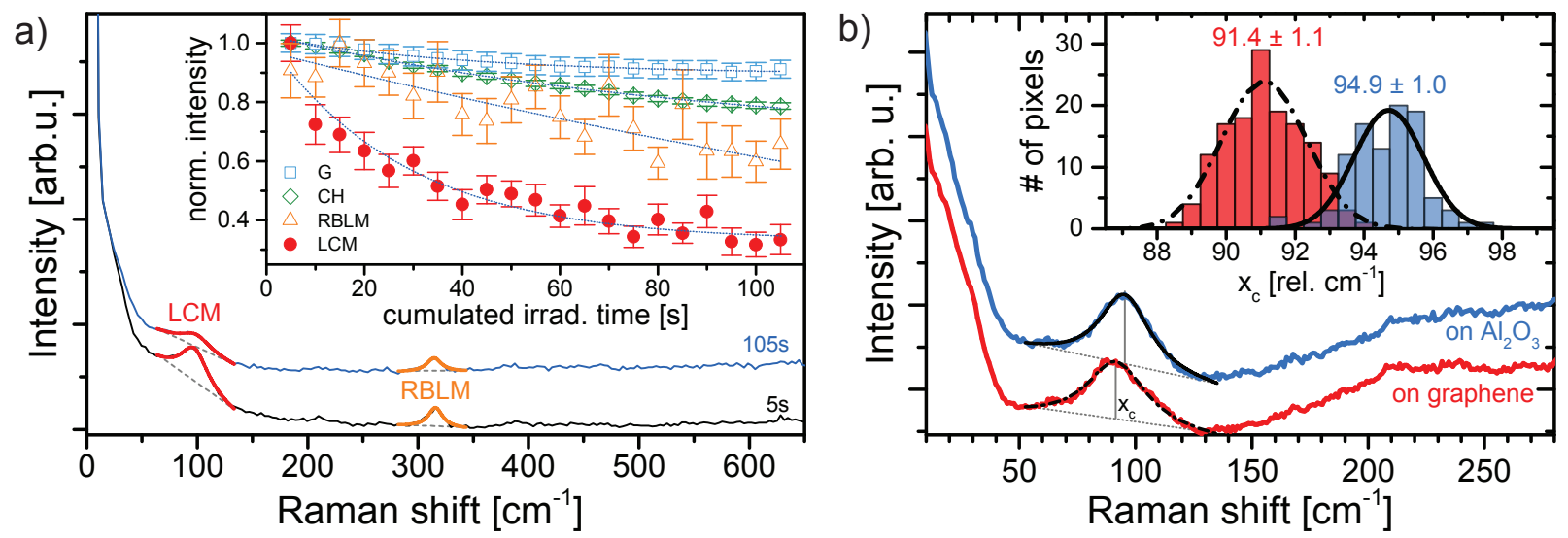

Figure 4: Damage monitoring and substrate sensitivity via the LCM-peak of 9AGNRs. a) Low energy spectra acquired with $5 \mathrm{~s}$ integration time and after an additional 100 s laser irradiation time at the same sample location, showing GNR damaging. The inset shows the normalized peak intensity obtained from a Lorentzian fit of the LCM, RBLM, CH$\&$ G-peaks as a function of the cumulated irradiation time. Full data in Figure S12, Dashed lines are fits with simple exponential decays. $\lambda_{\text {exc }}=488 \mathrm{~nm}, P=5 \mathrm{~mW}$, Sample 9-2_T. b) Substrate-dependent Raman shift of the LCM for GNRs on oxide or on a graphene interlayer. The inset shows histograms for the peak center extracted from a single Raman map containing both substrates, fitted with Gaussian distributions. $\lambda_{\mathrm{exc}}=488 \mathrm{~nm}$, Sample 9-1_T.

LCM as a tool to assess ribbon damage and substrate interaction. In addition to being useful to rapidly assess non-ideal growth regimes that result in short GNRs, the LCM is also a sensitive probe for ribbon integrity. The atomic displacement shown in Figure $1 \mathrm{c}$ ) shows that the LCM requires the coherent vibration of all atoms along the ribbon. It can therefore be expected that the presence of defects in GNRs as well as the interaction with the substrate on which the ribbon is placed has a strong influence on it, especially for long ribbons.

To systematically investigate damaging of GNRs, a transferred film of 9-AGNRs was irradiated with high laser power $(5 \mathrm{~mW})$ at $488 \mathrm{~nm}$, leading to the introduction of defects. ${ }^{31}$ Figure $4 \mathrm{a})$ shows spectra acquired on the same sample spot after short and prolonged laser exposure. The inset shows the fitted intensity of the LCM, RBLM, CH- and G-peaks as a function of the accumulated irradiation time by the excitation laser while acquiring the spectra. While all peaks show an intensity decrease with increasing irradiation time, this decay is most pronounced for the LCM. Figure S12 c, d) displays a power-dependent series 
of spectra, all normalized to a common power-time product. The LCM clearly disappears as the power and corresponding laser damage is increased. This shows that the LCM intensity is a good tool for monitoring the ribbon integrity throughout device processing and may be a key identifier for GNRs' suitability in optoelectronic applications.

Inspired by the long-range nature of this mode, we investigated whether there is any influence of the target substrate onto which the GNRs are transferred. Two technologically relevant substrates are oxide surfaces used for gate-insulation in field-effect transistors and graphene, which is increasingly used as an electrode material. 2132 We transferred a sample of 9-AGNRs on a patterned graphene-on-oxide substrate and acquired Raman spectra scanning over the boundary of both substrates (Figure S13). Interestingly, we find a strong down-shift of the LCM frequency for GNRs on graphene, a change of nearly 4\%. No comparable shift is observed for any of the other Raman modes of GNRs. Low-frequency modes have been used extensively over the last years to probe the layer interactions of $2 \mathrm{D}$-materials. $\frac{\sqrt[33]{35}}{3}$ The observation of a significant substrate-dependent effect on the LCM points towards a host of so far unexplored phenomena relevant for the fabrication of hybrid devices that can be explored via these low-frequency modes.

Matching calculated Raman spectra and experimental data. We find a good overall match between Raman spectra, first-principles calculations and independent observations from (low-temperature)-STM. The high background and low Raman intensity when measuring on gold, however, prevent us from observing the LCM in the samples containing on average the longest GNRs while still on the growth substrate (see Figure S9). Moreover, the frequency of the LCM observed after transfer to device substrates is substantially higher than that predicted by calculations for isolated GNRs, matching better with the overtone denoted LCM3. In contrast, for short 5-AGNRs the LCM is observed precisely at the frequencies predicted by DFT on both growth and device substrates. To address this discrepancy for long GNRs we focused on 9-AGNRs, which reproducibly show the LCM at $96 \mathrm{~cm}^{-1}$ for $\lambda_{\text {exc }}=$ $488 \mathrm{~nm}$, the wavelength for which our setup allows measurements at the lowest wavenumbers. 

$96 \mathrm{~cm}^{-1}$ but also of the background below about $60 \mathrm{~cm}^{-1}$. Our spectral resolution still does not allow us to discriminate whether this low-energy polarization dependent background is a signature of another lower-energy Raman peak or due to increased scattering of the tail of the laser from the ribbons, which should also follow the variation of the GNRs' interaction crosssection with polarization. To access the ultra-low spectral range predicted to contain the fundamental LCM for longer ribbons one would need to further reduce Rayleigh scattering and improve filtering. Selective synthesis of intermediate length $(5-7 \mathrm{~nm})$ GNRs would allow to find a definite answer. Based on the strong substrate-sensitivity shown in Figure $4 \mathrm{~b}$ ), we interpret the observed peak at $96 \mathrm{~cm}^{-1}$ as the LCM of 9-AGNRs being up-shifted in energy relative to the gas phase calculations due to the interaction with the substrate. In particular, we attribute this shift to localized pinning of the GNR on the rougher device substrate. This effect, which is not included in our calculations, can lead to a reduced effective length of freely vibrating GNR sections thereby up-shifting the modes towards the experimentally observed frequencies. The importance of modeling the substrate is further highlighted by its selective influence on longer GNRs. To accurately model an experimental spectrum, which includes contributions of typically hundreds of GNRs within the size of the laser spot, one can extract the length distribution of GNRs from high-resolution STM images and compute an accordingly weighted sum of calculated spectra (see Figure S14 in SI Note 5). This summation approach qualitatively reproduces the spectra, in particular the signatures of short ribbons and the observed increase in background intensity around $100 \mathrm{~cm}^{-1}$. The discrepancy with the experimental spectra is, again, attributed to the fact that we neglect the substrate effect in the calculations. We anticipate that the inclusion of such a substrate model will allow the reverse approach of deducing a quantitative length-distribution of GNRs in a sample by Raman spectroscopy alone. An improved understanding of substrate effects is the focus of an ongoing study and beyond the scope of this paper. 


\section{Conclusions}

We have identified and characterized a so-far unreported longitudinal vibrational mode in AGNRs by Raman spectroscopy. Its length-dependent Raman shift has been investigated by adjusting the GNR growth parameters and is supported by a combined analysis with high resolution STM and first-principles calculations. We demonstrated the usefulness of the LCM for the rapid identification of samples containing short ribbon-segments on both growth and device substrates. This information would otherwise require time-consuming and expensive low-temperature STM for probing the GNRs' length on the growth substrate and would not at all be accessible after GNR transfer and device integration. Moreover, we show the mode's sensitivity to damage of the GNRs. This makes it ideally suited to study the effects of processing and nano-fabrication on GNRs, which therefore has become a routine practice in our labs. Finally, we observe a particularly strong influence of the substrate on the vibrational frequency of this mode, pointing towards the possibility to investigate the interaction of GNRs with their environment by Raman spectroscopy. We anticipate that this mode will also be observed in GNRs with different edge structures and that these findings will substantially advance the overall understanding of GNRs, their interaction with diverse substrates, and provide insights that we deem crucial for the deterministic fabrication of GNR-based devices.

\section{Methods}

On surface synthesis and STM characterization of AGNRs. 5-AGNRs were synthesized from an isomeric mixture of 3,9-diiodoperylene and 3,10-diiodoperylene (DIP) as the precursor monomer - referred to as iodine-based. 5-AGNRs with bromine-based precursors were synthesized from an isomeric mixture of 3,9-dibromoperylene and 3,10-dibromoperylene (DBP). $\stackrel{36}{7-A G N R s ~ w e r e ~ s y n t h e s i z e d ~ f r o m ~ 10,10 '-d i b r o m o-9,9 '-b i a n t h r y l ~(D B B A) ~} 1$ and 9AGNRs from 3',6'-di-iodine-1,1':2',1"'terphenyl (DITP). ${ }^{24}$ First, Au(788) single crystals 
(Matec, Germany) or Au/mica substrates (Phasis, Switzerland) were cleaned in ultra-high vacuum by two sputtering/annealing cycles: $1 \mathrm{kV}$ Ar+ for 10 minutes followed by annealing at $470{ }^{\circ} \mathrm{C}$ for 10 minutes. Next, the monomer was sublimed onto the Au surface from a quartz crucible heated to $70{ }^{\circ} \mathrm{C}$ (DITP), $200{ }^{\circ} \mathrm{C}(\mathrm{DBBA})$, and $200^{\circ} \mathrm{C}(\mathrm{DBP} / \mathrm{DIP})$ respectively, with the substrate held at room temperature. After 1 monolayer coverage deposition, for both 7-, and 9-AGNRs the substrate was heated $(0.5 \mathrm{~K} / \mathrm{s})$ up to $200^{\circ} \mathrm{C}$ with a 10 minute holding time to activate the polymerization reaction, followed by annealing at $400^{\circ} \mathrm{C}(0.5 \mathrm{~K} / \mathrm{s}$ with a 10 minute holding time) in order to form the GNRs via cyclodehydrogenation. For the synthesis of the 5-AGNRs a slow annealing $(0.2 \mathrm{~K} / \mathrm{s})$ was carried up to $225^{\circ} \mathrm{C}$.

Topographic scanning tunneling microscopy images of as-grown AGNRs on $\mathrm{Au}(788)$ and $\mathrm{Au} /$ mica were taken with a Scienta Omicron VT-STM/LT-STM operated at room temperature $/ 5 \mathrm{~K}$. Constant-current STM images were recorded with $-1.5 \mathrm{~V}$ sample bias and $0.03 \mathrm{nA}$ setpoint current. The length analysis of the ribbons was performed in Igor Pro using an image processing script developed in-house. In total we identified 123 ribbons for the histogram in Figure S2 and 261 ribbons for the histogram in Figure S3. By counting GNRs in different scan images, we ensure that this is a representative length distribution of these samples.

Transfer of GNRs to substrates. AGNRs were transferred from their Au/mica or $\mathrm{Au}(788)$ growth substrate to silicon-based substrates by two different transfer approaches. $\mathrm{Au} /$ mica samples were transferred using a polymer-free method described elsewhere. ${ }^{12}$ Samples grown on $\mathrm{Au}(788)$ crystals were transferred by an electrochemical delamination method. First, PMMA was spin-coated (2500 rpm for 90 seconds, 4 layers) on GNR/Au to act as a support layer during the transfer, followed by a 10 minutes curing step at $80^{\circ} \mathrm{C}$. In order to decrease the PMMA delamination time, the PMMA on the edges of the Au (788) crystal was removed after UV-exposure ( 80 minutes) followed by 3 minutes development in water/isopropanol. The electrochemical delamination transfer was carried out using $1 \mathrm{M}$ $\mathrm{NaOH}$ aqueous solution as electrolyte. The electrochemical cell was mounted using a carbon 
rod as anode and the PMMA/GNR/Au as the cathode. A DC voltage of 5V was applied between anode and cathode for 1 minute (resulting current around 0.2 A). Hydrogen bubbles form at the interface of PMMA/GNR and Au, resulting in delamination of the PMMA/GNR layer. The PMMA/GNR layer was cleaned for 5 minutes in purified water before being transferred to the target substrate. Subsequently, the PMMA/GNR/substrate stack was cured for 10 minutes at $80^{\circ} \mathrm{C}$ followed by 20 minutes at $110^{\circ} \mathrm{C}$ to increase the adhesion between the target substrate and the PMMA/GNR layer. Finally, the PMMA was dissolved in acetone (15 minutes) followed by an ethanol-rinse.

Raman spectroscopy experiments. Raman spectra were acquired with a WITec Alpha $300 R$ confocal Raman microscope in backscattering geometry. The linear polarization of the exciting lasers was adjusted with a motorized $\lambda / 2$ plate. Rayleigh scattered light is blocked by a notch filter (specifically, a volume holographic grating, referred to as 'Ray-shield'-option) for $488 \mathrm{~nm}$ excitation and edge filters for $532 \mathrm{~nm} / 785 \mathrm{~nm}$ excitation. The backscattered light was filtered with an analyzing polarizer only where explicitly stated and coupled to one of two spectrometers: a $300 \mathrm{~mm}$ lens-based spectrometer with gratings of $600 \mathrm{~g} / \mathrm{mm}$ or $1800 \mathrm{~g} / \mathrm{mm}$ equipped with a thermoelectrically cooled CCD for $488 \mathrm{~nm}$ and $532 \mathrm{~nm}$ excitation and a $400 \mathrm{~mm}$ lens-based spectrometer with gratings of $300 \mathrm{~g} / \mathrm{mm}$ or $1200 \mathrm{~g} / \mathrm{mm}$ equipped with a cooled deep-depletion CCD for $785 \mathrm{~nm}$ excitation. The laser wavelength, power and integration time were optimized for each type of GNR and substrate to maximize the signal. $\frac{22}{22}$ The sample was mounted in a home-built vacuum chamber at a pressure below $10^{-2}$ mbar. For maximum signal intensity spectra are recorded with a Zeiss 50x LD objective, $\mathrm{NA}=0.55$, through an uncoated fused silica window of only $0.2 \mathrm{~mm}$ thickness covering a hole of just $7 \mathrm{~mm}$ diameter. The chamber is mounted on a piezo stage for scanning. A polynomial background was subtracted from the raw spectra to remove signatures of photoluminescence (see Figure S6]. 31

Computational methods. Normal modes and Raman intensities were calculated using density functional theory (DFT) for small finite size and periodic GNRs, and a combination 


\section{Author contributions}

J.O. performed Raman measurements; J.O., G.B.B., M.L.P., M.D.L. and M.C. analyzed the Raman results; C.D. performed Raman calculations; G.B.B. optimized the GNR growth; G.B.B, R.D. and Q.S performed on-surface synthesis and STM imaging of GNR; J.O., O.B. fabricated device substrates and with G.B.B. and R.D. optimized the GNR transfer; XY.W. and T.D. synthesized the precursor molecules under supervision of A.N. and K.M.; J.O, G.B.B. and M.L.P. wrote the manuscript; P.R., V.M., R.F. and M.C. supervised the work. All authors commented on the manuscript. 


\section{Acknowledgement}

This work was supported by the Swiss National Science Foundation under grant no. 20PC21_155644 and the NCCR MARVEL (51NF40_182892), the European Union's Horizon 2020 research and innovation program under grant agreement no. 785219 (Graphene Flagship Core 2) and grant agreement no. 767187 (QuIET), and the Office of Naval Research (N00014-18-1-2708 and N00014-12-1-1009). Part of the computations were performed using resources of the Center for Computational Innovation at Rensselaer Polytechnic Institute. M.L.P. acknowledges funding by the EMPAPOSTDOCS-II program, which is financed by the European Union's Horizon 2020 research and innovation program under the Marie Sklodowska-Curie grant agreement no. 754364. M.D.L. acknowledges support from the Swiss National Science Foundation Ambizione grant (no. PZ00P2_179801). X. Y. W., T. D., A. N., and K. M. acknowledge the support by the Max Planck Society. J.O. and O.B. acknowledge technical support from the Binning and Rohrer Nanotechnology Center (BRNC), J.O. and M.C. thank R. Furrer, R. Muff, S. Martin, H. Breitenstein for technical support. The authors thank M. El Abbassi, M. Di Giovannantonio, and I. Zardo for fruitful discussions and E. Hack for carefully reading the manuscript.

\section{Supporting Information Available}

The Supporting Information is available free of charge on the ACS Publications website.

1. Experimental details on sample fabrication and STM data

2. A theoretical discussion of the fundamental origin of the LCM in finite-size GNRs

3. DFT-calculated mode profiles

4. Additional Raman data and reference measurements 
5. Additional data on the damage sensitivity, substrate interaction and length distribution assessment of GNRs

\section{References}

1. Cai, J.; Ruffieux, P.; Jaafar, R.; Bieri, M.; Braun, T.; Blankenburg, S.; Muoth, M.; Seitsonen, A. P.; Saleh, M.; Feng, X.; Müllen, K.; Fasel, R. Atomically Precise BottomUp Fabrication of Graphene Nanoribbons. Nature 2010, 466, 470-473.

2. Kimouche, A.; Ervasti, M. M.; Drost, R.; Halonen, S.; Harju, A.; Joensuu, P. M.; Sainio, J.; Liljeroth, P. Ultra-Narrow Metallic Armchair Graphene Nanoribbons. Nat. Commun. 2015, 6, 10177.

3. Talirz, L.; Söde, H.; Dumslaff, T.; Wang, S.; Sanchez-Valencia, J. R.; Liu, J.; Shinde, P.; Pignedoli, C. A.; Liang, L.; Meunier, V.; Plumb, N. C.; Shi, M.; Feng, X.; Narita, A.; Müllen, K.; Fasel, R.; Ruffieux, P. On-Surface Synthesis and Characterization of 9-Atom Wide Armchair Graphene Nanoribbons. ACS Nano 2017, 11, 1380-1388.

4. Schwierz, F. Graphene Transistors. Nat. Nanotechnol. 2010, 5, 487-496.

5. Fujita, M.; Wakabayashi, K.; Nakada, K.; Kusakabe, K. Peculiar Localized State at Zigzag Graphite Edge. J. Phys. Soc. Jpn. 1996, 65, 1920-1923.

6. Son, Y.-W.; Cohen, M. L.; Louie, S. G. Energy Gaps in Graphene Nanoribbons. Phys. Rev. Lett. 2006, 97, 216803.

7. Chen, Y.-C.; de Oteyza, D. G.; Pedramrazi, Z.; Chen, C.; Fischer, F. R.; Crommie, M. F. Tuning the Band Gap of Graphene Nanoribbons Synthesized from Molecular Precursors. ACS Nano 2013, 7, 6123-6128.

8. Ruffieux, P.; Wang, S.; Yang, B.; Sánchez-Sánchez, C.; Liu, J.; Dienel, T.; Talirz, L.; Shinde, P.; Pignedoli, C. A.; Passerone, D.; Dumslaff, T.; Feng, X.; Müllen, K.; Fasel, R. 
On-Surface Synthesis of Graphene Nanoribbons with Zigzag Edge Topology. Nature 2016, 531, 489-492.

9. Gröning, O.; Wang, S.; Yao, X.; Pignedoli, C. A.; Borin Barin, G.; Daniels, C.; Cupo, A.; Meunier, V.; Feng, X.; Narita, A.; Müllen, K.; Ruffieux, P.; Fasel, R. Engineering of Robust Topological Quantum Phases in Graphene Nanoribbons. Nature 2018, 560, 209213.

10. Rizzo, D. J.; Veber, G.; Cao, T.; Bronner, C.; Chen, T.; Zhao, F.; Rodriguez, H.; Louie, S. G.; Crommie, M. F.; Fischer, F. R. Topological Band Engineering of Graphene Nanoribbons. Nature 2018, 560, 204-208.

11. Fairbrother, A.; Sanchez-Valencia, J.-R.; Lauber, B.; Shorubalko, I.; Ruffieux, P.; Hintermann, T.; Fasel, R. High Vacuum Synthesis and Ambient Stability of Bottom-Up Graphene Nanoribbons. Nanoscale 2017, 9, 2785-2792.

12. Borin Barin, G.; Fairbrother, A.; Rotach, L.; Bayle, M.; Paillet, M.; Liang, L.; Meunier, V.; Hauert, R.; Dumslaff, T.; Narita, A.; Müllen, K.; Sahabudeen, H.; Berger, R.; Feng, X.; Fasel, R.; Ruffieux, P. Surface-Synthesized Graphene Nanoribbons for Room Temperature Switching Devices: Substrate Transfer and Ex Situ Characterization. ACS Appl. Nano Mater. 2019, 2, 2184-2192.

13. Llinas, J. P.; Fairbrother, A.; Borin Barin, G.; Shi, W.; Lee, K.; Wu, S.; Yong Choi, B.; Braganza, R.; Lear, J.; Kau, N.; Choi, W.; Chen, C.; Pedramrazi, Z.; Dumslaff, T.; Narita, A.; Feng, X.; Müllen, K.; Fischer, F.; Zettl, A.; Ruffieux, P. et al. Short-Channel Field-Effect Transistors with 9-Atom and 13-Atom Wide Graphene Nanoribbons. Nat. Commun. 2017, 8, 633 .

14. Chong, M. C.; Afshar-Imani, N.; Scheurer, F.; Cardoso, C.; Ferretti, A.; Prezzi, D.; Schull, G. Bright Electroluminescence from Single Graphene Nanoribbon Junctions. Nano Lett. 2017, 18, 175-181. 
15. Gillen, R.; Mohr, M.; Thomsen, C.; Maultzsch, J. Vibrational Properties of Graphene Nanoribbons by First-Principles Calculations. Phys. Rev. B 2009, 80, 155418.

16. Gillen, R.; Mohr, M.; Maultzsch, J. Symmetry Properties of Vibrational Modes in Graphene Nanoribbons. Phys. Rev. B 2010, 81, 205426.

17. Vandescuren, M.; Hermet, P.; Meunier, V.; Henrard, L.; Lambin, P. Theoretical Study of the Vibrational Edge Modes in Graphene Nanoribbons. Phys. Rev. B 2008, 78, 195401.

18. Verzhbitskiy, I. A.; Corato, M. D.; Ruini, A.; Molinari, E.; Narita, A.; Hu, Y.; Schwab, M. G.; Bruna, M.; Yoon, D.; Milana, S.; Feng, X.; Müllen, K.; Ferrari, A. C.; Casiraghi, C.; Prezzi, D. Raman Fingerprints of Atomically Precise Graphene Nanoribbons. Nano Lett. 2016, 16, 3442-3447.

19. Zhou, J.; Dong, J. Vibrational Property and Raman Spectrum of Carbon Nanoribbon. Appl. Phys. Lett. 2007, 91, 173108.

20. Chen, Z.; Wang, H. I.; Bilbao, N.; Teyssandier, J.; Prechtl, T.; Cavani, N.; Tries, A.; Biagi, R.; de Renzi, V.; Feng, X.; Kläui, M.; de Feyter, S.; Bonn, M.; Narita, A.; Müllen, K. Lateral Fusion of Chemical Vapor Deposited N $=5$ Armchair Graphene Nanoribbons. J. Am. Chem. Soc. 2017, 139, 9483-9486.

21. Martini, L.; Chen, Z.; Mishra, N.; Borin Barin, G.; Fantuzzi, P.; Ruffieux, P.; Fasel, R.; Feng, X.; Narita, A.; Coletti, C.; Müllen, K.; Candini, A. Structure-Dependent Electrical Properties of Graphene Nanoribbon Devices with Graphene Electrodes. Carbon 2019, $146,36-43$.

22. Overbeck, J.; Borin Barin, G.; Daniels, C.; Perrin, M.; Liang, L.; Braun, O.; Darawish, R.; Burkhardt, B.; Dumslaff, T.; Wang, X.-Y.; Narita, A.; Müllen, K.; Meunier, V.; Fasel, R.; Calame, M.; Ruffieux, P. Optimized Substrates and Measurement Approaches for Raman Spectroscopy of Graphene Nanoribbons. arXiv e-prints 2019, http://arxiv.org/abs/1907.01797 (accessed Sept. 19, 2019). 
23. Ma, C.; Liang, L.; Xiao, Z.; Puretzky, A. A.; Hong, K.; Lu, W.; Meunier, V.; Bernholc, J.; Li, A.-P. Seamless Staircase Electrical Contact to Semiconducting Graphene Nanoribbons. Nano Lett. 2017, 17, 6241-6247.

24. Di Giovannantonio, M.; Deniz, O.; Urgel, J. I.; Widmer, R.; Dienel, T.; Stolz, S.; Sánchez-Sánchez, C.; Muntwiler, M.; Dumslaff, T.; Berger, R.; Narita, A.; Feng, X.; Müllen, K.; Ruffieux, P.; Fasel, R. On-Surface Growth Dynamics of Graphene Nanoribbons: The Role of Halogen Functionalization. ACS Nano 2018, 12, 74-81.

25. Medina Rivero, S.; Canola, S.; Zeng, W.; Ramírez, F. J.; Zafra, J. L.; Wu, J.; Negri, F.; Casado, J. Long Rylene Nanoribbons Express Polyacetylene-Like Signatures at their Edges. Phys. Chem. Chem. Phys. 2019, 21, 7281-7287.

26. Gillen, R.; Mohr, M.; Maultzsch, J. Raman-Active Modes in Graphene Nanoribbons. Phys. Status Solidi B 2010, 247, 2941-2944.

27. Gommans, H. H.; Alldredge, J. W.; Tashiro, H.; Park, J.; Magnuson, J.; Rinzler, A. G. Fibers of Aligned Single-Walled Carbon Nanotubes: Polarized Raman Spectroscopy. J. Appl. Phys. (Melville, NY, U. S.) 2000, 88, 2509-2514.

28. Wang, J.; Gudiksen, M. S.; Duan, X.; Cui, Y.; Lieber, C. M. Highly Polarized Photoluminescence and Photodetection from Single Indium Phosphide Nanowires. Science 2001, 293, 1455-1457.

29. Jorio, A.; Souza Filho, A. G.; Brar, V. W.; Swan, A. K.; Ünlü, M. S.; Goldberg, B. B.; Righi, A.; Hafner, J. H.; Lieber, C. M.; Saito, R.; Dresselhaus, G.; Dresselhaus, M. S. Polarized Resonant Raman Study of Isolated Single-Wall Carbon Nanotubes: Symmetry Selection Rules, Dipolar and Multipolar Antenna Effects. Phys. Rev. B 2002, 65, 121402.

30. Saito, R.; Furukawa, M.; Dresselhaus, G.; Dresselhaus, M. S. Raman Spectra of Graphene Ribbons. J. Phys.: Condens. Matter 2010, 22, 334203. 
31. Senkovskiy, B. V.; Pfeiffer, M.; Alavi, S. K.; Bliesener, A.; Zhu, J.; Michel, S.; Fedorov, A. V.; German, R.; Hertel, D.; Haberer, D.; Petaccia, L.; Fischer, F. R.; Meerholz, K.; van Loosdrecht, P. H. M.; Lindfors, K.; Grüneis, A. Making Graphene Nanoribbons Photoluminescent. Nano Lett. 2017, 17, 4029-4037.

32. El Abbassi, M.; Sangtarash, S.; Liu, X.; Perrin, M. L.; Braun, O.; Lambert, C.; van der Zant, H. S. J.; Yitzchaik, S.; Decurtins, S.; Liu, S.-X.; Sadeghi, H.; Calame, M. Robust Graphene-Based Molecular Devices. Nat. Nanotechnol. 2019, in press.

33. Tan, P. H.; Han, W. P.; Zhao, W. J.; Wu, Z. H.; Chang, K.; Wang, H.; Wang, Y. F.; Bonini, N.; Marzari, N.; Pugno, N.; Savini, G.; Lombardo, A.; Ferrari, A. C. The Shear Mode of Multilayer Graphene. Nat. Mater. 2012, 11, 294-300.

34. Huang, S.; Liang, L.; Ling, X.; Puretzky, A. A.; Geohegan, D. B.; Sumpter, B. G.; Kong, J.; Meunier, V.; Dresselhaus, M. S. Low-Frequency Interlayer Raman Modes to Probe Interface of Twisted Bilayer MoS2. Nano Lett. 2016, 16, 1435-1444.

35. Liang, L.; Puretzky, A. A.; Sumpter, B. G.; Meunier, V. Interlayer Bond Polarizability Model for Stacking-Dependent Low-Frequency Raman Scattering in Layered Materials. Nanoscale 2017, 9, 15340-15355.

36. Schlichting, P.; Rohr, U.; Müllen, K. New Synthetic Routes to Alkyl-Substituted and Functionalized Perylenes. Liebigs Ann. 1997, 1997, 395-407.

37. Kresse, G.; Hafner, J. Ab Initio Molecular Dynamics for Liquid Metals. Phys. Rev. B 1993, $47,558-561$.

38. Kresse, G.; Furthmüller, J. Efficiency of Ab-Initio Total Energy Calculations for Metals and Semiconductors Using a Plane-Wave Basis Set. Comput. Mater. Sci. 1996, 6, 15-50.

39. Kresse, G.; Furthmüller, J. Efficient Iterative Schemes for Ab Initio Total-Energy Calculations Using a Plane-Wave Basis Set. Phys. Rev. B 1996, 54, 11169-11186. 
40. Kresse, G.; Joubert, D. From Ultrasoft Pseudopotentials to the Projector AugmentedWave Method. Phys. Rev. B 1999, 59, 1758-1775.

41. Perdew,; Burke,; Ernzerhof, Generalized Gradient Approximation Made Simple. Phys. Rev. Lett. 1996, 777, 3865-3868.

42. Long, D. A. The Raman Effect; John Wiley \& Sons, Ltd: Chichester, UK, 2002.

43. Gajdoš, M.; Hummer, K.; Kresse, G.; Furthmüller, J.; Bechstedt, F. Linear Optical Properties in the Projector-Augmented Wave Methodology. Phys. Rev. B 2006, 73, 045112.

44. Liang, L.; Meunier, V. First-Principles Raman Spectra of MoS2, WS2 and their Heterostructures. Nanoscale 2014, 6, 5394-5401.

45. Brenner, D. W.; Shenderova, O. A.; Harrison, J. A.; Stuart, S. J.; Ni, B.; Sinnott, S. B. A Second-Generation Reactive Empirical Bond Order (REBO) Potential Energy Expression for Hydrocarbons. J. Phys.: Condens. Matter 2002, 14, 783-802.

46. Nakadaira, M.; Saito, R.; Kimura, T.; Dresselhaus, G.; Dresselhaus, M. S. Excess Li Ions in a Small Graphite Cluster. J. Mater. Res. 1997, 12, 1367-1375.

47. Togo, A.; Tanaka, I. First Principles Phonon Calculations in Materials Science. Scr. Mater. 2015, 108, 1-5. 


\section{Graphical TOC Entry}

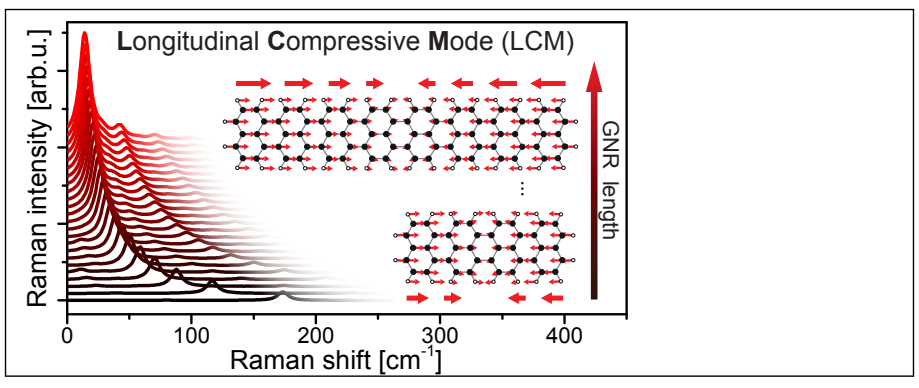

15

16

17

18

19

20

21

22

23

24

25

26

27

28

29

30

31

32

33

34

35

36

37

38

39

40

41

42

43

44

45

46

47

48

49

50

51

52

53

54

55

56

57

58

59

60

ACS Paragon Plus Environment 